As to how the disease was first introduced there is some doubt. It is generally believed that the bacillus suisepticus may exist as a harmless saprophyte, and from apparently healthy swine certain authorities have isolated organisms bearing a close resemblance in many respects with the bacillus suisepticus, but not, under ordinary conditions, virulent for swine on experimental inoculation. It would seem, however, that in the body of swine enfeebled through some adverse influence the bacillus suisepticus may acquire pathogenic properties.

There is little doubt that this is the manner in which swine-plague occurs as a complication of swine-fever, the filtrable virus of the latter serving to lower the natural resistance of swine against the harmful effect of the swine-plague bacillus.

In the case of the above-described outbreak a number (twelve) of pigs had been purchased and introduced on the farm some three months prior to the occurrence of the heavy mortality, and although these animals came from, so far as is known, a non-infected area, one died soon after their arrival on the new premises. This was followed by one death after another amongst the recently introduced pigs, until finally only four of them remained, when the heavy mortality commenced amongst the young (and presumably very susceptible) pigs born on the farm since the arrival of the twelve purchased ones.

Beyond this it was impossible to trace the source of infection. However, the four pigs remaining alive out of the purchased lot were destroyed, as it was feared they might act as "carriers" of the virus. The premises were also disinfected, with the result that, as far as the swine remaining on this particular farm were concerned, the mortality promptly ceased.

\title{
EPIZOOTIC PNEUMO-PERICARDITIS IN THE TURKEY *
}

By Walter JowetT, F.R.C.V.S., D.V.H., Department of Agriculture, Cape Town.

THE following observations relate to an infective disease in the turkey, characterised by pneumo-pericardial lesions.

It is now some years since M'Fadyean ${ }^{1}$ first described this disease in England, and the available veterinary literature contains no other account of the malady. The writer's attention was first drawn to this particular disease by the owner of a large flock of turkeys, situated some miles from Cape Town. This gentleman reported the occurrence amongst his birds of a mysterious disease which had carried off some twenty-seven out of the hundred or so turkeys in his possession. In due course a carcase arrived for examination :-

Turkey No. I was a "bronze" male bird in good condition. On examination attention was at once attracted by the presence of severe pericarditis. The pericardium had contracted extensive

I "Journal of Comparative Pathology and Therapeutics," Vol. VI., p. 334, 1893. 
adhesions with the heart wall, which was entirely covered with a layer of yellow-coloured fibrinous exudate. The right lung was completely hepatised, the left hyperæmic. The liver was somewhat fatty, and the kidneys seemed pale and swollen, but no marked changes were apparent in any other organs. Smears from the heart blood, pericardial exudate, spleen, lung, and liver were stained and submitted to microscopical examination, with the result that a small bipolar-staining bacterium was everywhere found present. Bouillon and agar tubes inoculated from the heart blood and surface of heart yielded a pure culture.

A visit was next paid to the infected premises and three turkeys

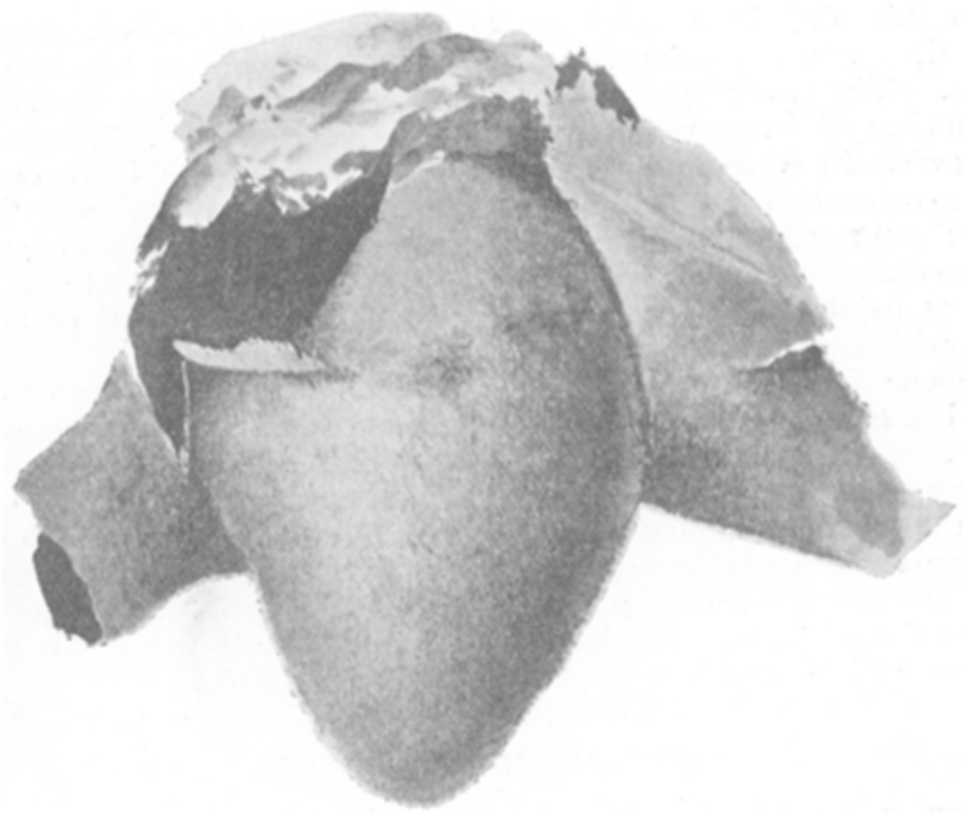

Fic. 1.

Heart of turkey No. 1, showing fibrinots exulate on surface.

which had recently succumbed were submitted to examination. The post-mortem findings were as follows:-

Turkey No. 2.- A young cock bird in good condition. On opening the abdominal cavity a quantity of blood-stained fluid was noticed. but the abdominal viscera seemed quite healthy in appearance, There was severe pericarditis, a quantity of sero-fibrinous exudate being present in the pericardial sac. The right lung showed a few hepatised areas, whilst the left was merely hyperamic. Smears from the blood, spleen, lungs, and pericardial exudate revealed, on microscopical examination, the presence of the ovoid bacterium, especially numerous in the preparation from the two last-mentioned organs.

Turkey No. 3.- Young male bird in fair condition. Abdominal 
organs normal with the exception of the liver, which was distinctly fatty. Some post-morten discoloration of abdominal viscera. Pericardial sac contained a quantity of serous fluid. No adhesions, no fibrinous exudate. The lungs were extensively congested, whilst the pleura showed early inflammatory changes. Smears from the heart blood, pericardial effusion, and lungs disclosed the presence of innumerable small ovoid, bipolar-staining bacteria.

Turkey' No. 4.-An imported "bronze" male, full grown, fair condition. A serous exudate present in the pericardial sac; both lungs hyperemic. In smears from heart blood, lungs, and pericardial effusion the small ovoid bacterium was detected, very plentiful in those from the lungs and pericardium, somewhat scanty in the blood smears.

In this bird, in addition to the lesions just described, attention was drawn to the abnormal appearance of the liver and the two creca. The liver was somewhat enlarged, and on its surface a number of firm round and oval areas were noticed. These were yellowish in colour, semi-solid, and extended some distance into the substance of the organ. On section similar areas were found to exist throughout the liver. The caca were enormously distended, their walls thickened, the mucous membrane covered with a soft, yellow gelatinous exudate, whilst the contents consisted of a soft, yellowish-white, pasty material.

Smears from the liver lesions examined for the presence of acidfast bacteria yielded negative results; finally the lesions were identified as those due to infectious entero-hepatitis, a disease seemingly not uncommon amongst "bronze" turkeys imported to this Colony from the United States of America. These last-mentioned lesions showed evidence of some chronicity, and presumably this particular bird had suffered from the effects of entero-hepatitis for some little time prior to death. The immediate cause of death was undoubtedly the complication with epizootic pneumo-pericarditis.

\section{INOCULATION EXPERIMENTS.}

Turkey' No. 5--Young cock turkey, in fair condition, procured together with the next bird, turkey No. 6, from a perfectly healthy flock. Inoculated subcutaneously in the pectoral region with I cc. of a pure bouillon culture of third generation. This culture was originally obtained from the heart blood of turkey No. I. About eighteen hours after inoculation the bird appeared sick, crouching in one position, feathers ruffled, and all food refused. Found dead forty-eight hours after inoculation.

Post-mortem. - At the point of inoculation in the pectoral region there was a dry necrotic area extending some little distance into the muscular tissue; films from this area were found simply teeming with the ovoid bacterium. The pericardium presented evidence of well marked inflammatory changes, there being present in the sac a quantity of sero-fibrinous exudate. Slight adhesions had formed between the heart wall and pericardium, especially well marked near the apex of the heart. The lungs were hyperæmic, but otherwise showed no very marked changes. The liver was pale and swollen; all other organs appeared quite normal. The 
ovoid bacterium was found in blood smears, and was very plentiful indeed in smears from the pericardial exudate and lungs. Pure bouillon and agar cultures were obtained from all three positions.

Turkey No. 6. - Young brown cock bird in fair condition. Received subcutaneously in pectoral region $I \mathrm{cc}$. of emulsion in sterile water of lymph flakes from pericardial exudate of turkey No. 2. Eighteen hours after inoculation this turkey was obviously ill; crouching, refused food, feathers ruffled, finally mouth open and oral breathing. Forty hours after inoculation found dead.

Post-mortem. - At site of inoculation the subcutaneous tissue was pale and necrotic. Abdominal organs normal. A quantity of serous exudate was. found in the pericardial sac, and the lungs were extensively congested. Films from the surface of the heart showed very many ovoid bacteria, and films from the lungs were similar, whilst in the blood smears the organism was also present. Inoculated on agar and in bouillon, pure cultures were obtained from the heart blood and pericardial exudate.

Fow'l No. I.- Inoculated intraperitoneally with I cc. of pure twentyfour-hours-old bouillon culture of ovoid bacterium isolated from heart blood of turkey No. I. Next day the bird appeared rather ill, remained in one position on floor of cage, feathers ruffled, and drowsy, but after three or four days seemed to recover. Finally, ten days after inoculation, the fowl was killed.

On post-mortem examination the carcase was emaciated, and a quantity of fibrinous exudate was found in the peritoneal cavity, glueing together the coils of intestine; the peritoneal membrane was not markedly reddened. No other changes were found in any other portion of the carcase; and the small ovoid bacterium could not be detected in smears from the surface of intestines, heart, or spleen, neither was it seen in smears from the heart blood or lungs. Bouillon and agar tubes were inoculated from the sites mentioned. All the tubes remained sterile with the exception of the one inoculated from the exudate on the surface of the intestines. This yielded a culture of the ovoid bacterium.

Focol No. 2.-Received $2 \mathrm{cc}$. of pure bouillon culture from turkey No. 6. Next day a small swelling was noticed at site of inoculation. Bird seemed in quite normal health, and experienced no ill effects from inoculation.

Pigeon No. I.--Inoculated intraperitoneally with $0.5 \mathrm{cc}$. twentyfour-hours-old bouillon culture of bacterium isolated from surface of heart of turkey No. 3. Found dead twenty hours later. Serofibrinous exudate in peritoneal cavity; no change in lungs, surface of heart, or pericardium. Films from blood contained innumerable ovoid bacteria in pure culture.

Pigeon No. 2.- Inoculated subcutaneously with I cc. of a fortyeight-hours-old pure bouillon culture of ovoid bacterium obtained from heart blood of turkey No. 4. Next day appeared drowsy and sick. Found dead forty-eight hours after inoculation. Subcutaneous tissue at site of inoculation pale and necrotic, excess of fluid in pericardial sac, blood dark and fluid. Ovoid bacteria very plentiful in blood smears. The halteridium was also present in the erythrocytes of this bird in very considerable numbers, but this organism is often found in the blood of pigeons examined here. Inoculated on 
slant agar and incubated at $37^{\circ} \mathrm{C}$, the heart blood yielded a pure culture of the ovoid bacterium.

Pigeon No. 3.-Received I cc. of twenty-four-hours-old bouillon culture, third generation from guinea-pig, subcutaneously in the pectoral region.

Result.-Negative.

Guiner-pig No. I.-White full-grown male inoculated intraperitoneally with $0.5 \mathrm{cc}$. blood of turkey No. I. Found dead eighteen hours later. The peritoneal cavity contained a considerable quantity of effusion, in smears from which the ovoid organism was easily demonstrated on microscopic examnation. The same organism was also present in the blood smears.

Guinea-pig No. 2.-Inoculated intraperitoneally with $0.5 \mathrm{cc}$. twentyfour-hours-old bouillon culture of organism isolated from surface of heart of turkey No. I. Found dead twenty hours later.

Post-mortem.-Quantity of exudate in peritoneal cavity; ovoid organism present in this in immense numbers and same organism noticed in blood smears.

Guinea-pig No. 3.- Inoculated subcutaneously with I cc. of fortyeight-hours-old bouillon culture of ovoid organism isolated from blood of turkey No. 4. Noticed ill eighteen hours later, and found dead twenty-four hours after inoculation.

Post-mortem.-Small abscess at point of inoculation surrounded by extensive subcutaneous oedema. Large intestines showed few submucous hæmorrhages. Abdominal and thoracic viscera otherwise normal in appearance. Blood dark and fluid; ovoid organism present in pure culture.

White Rat No. I.-Inoculated intraperitoneally with $0.5 \mathrm{cc}$. twentyfour-hours-old culture of ovoid organism from turkey. Found dead twenty hours later. No marked lesions found on post-mortem; blood was fluid and contained the ovoid organism in pure culture.

White Rat No. 2.- Inoculated subcutaneously with I cc. of fortyeight-hours-old bouillon culture from turkey. Next day dull and slightly ill. Abscess formed at point of inoculation. Rat finally recovered.

Rabbits.-Rabbits were not available for the purpose of experimental inoculation. M'Fadyean, however, found the organism markedly pathogenic for this animal.

\section{DESCRIPTION OF TIIE CAUSAL ORGANISM.}

As already mentioned, the causal micro-organism of the abovedescribed disease is a minute ovoid bacterium bearing a close morphological resemblance to the bacillus choleræe gallinarum.

Staining Reactions. - The organism is Gram negative, but stains readily with aqueous solutions of the ordinary aniline dyes, exhibiting bipolar staining; hence, depending upon the degree of staining, the organism presents the appearance either of a "coccobacillus," "vacuole" bacillus, or a figure-of-eight form. Moreover, it is distinctly polymorphous.

Cultivation on Gelatine.-No liquefaction.

Streak Cultures. - The organism develops in small translucent colonies, which do not spread far from the track of the needle. 
Stab Cultures.-A distinctly visible growth along the track of the needle, composed of small circular colonies.

On Agar Slant.--Small, thin, translucent circular colonies form after twenty-four hours' incubation at $37^{\circ} \mathrm{C}$. These later become granular and more opaque (and in some cultures present a slightly white appearance), but even after some days incubation the growth is merely a thin granular film, perceptible only when the tube is closely examined. It is often quite difficult to detach the growth from the surface of the culture medium by means of the platinum needle.

Agar Shake. - Small discrete white colonies form; no gas formation.

Bouillon.-Uniformly turbid after twenty-four hours' incubation at $37^{\circ} \mathrm{C}$.; later a sediment forms, the upper portion of the medium clearing to a certain extent.

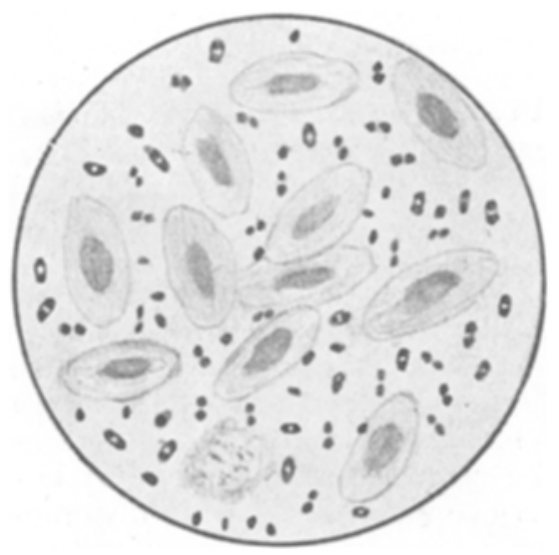

Fig. 2.

Blood smear from experimentally infected turkey. Eosin and methylene-blue stain $(\times 1000)$.

Potato.-No visible growth.

Litmus Milk.-No acid. No coagulation.

The organism is readily cultivated on agar or bouillon under either ærobic or anærobic conditions. On prolonged cultivation on the usual laboratory culture media the rirulence soon becomes attenuated.

Pathogenesis. - In turkeys after subcutaneous inoculation with pure cultures of the organism there are symptoms of dulness, stiffness, and oral breathing, followed by death in two or three days. At autopsy, pneumonia and pericarditis are found. The organism is abundantly present in the lungs and pericardial exudate. It is also present in the blood. The organism is virulent for guinea-pigs, and, according to $M$ Fadyean, it is even more virulent for the rabbit. Fowls are only slightly susceptible, pigeons succumb to intraperitoneal inoculation and also to subcutaneous, but for these birds the results are rather uncertain.

The white rat may be infected by the intraperitoneal injection of 
pure cultures, but in one experiment the same culture produced little effect by subcutaneous inoculation.

Fowls and geese appear to possess a natural immunity.

In the present outbreak, fowls, geese, and pigeons were in close contact with the diseased turkeys, and yet only the turkeys succumbed. In this respect the disease differs markedly from fowl-cholera; moreover, in the last-mentioned disease the lesions of pneumonia and pericarditis are not commonly encountered-at any rate not in the same degree or to the same extent as in the disease above described.

In the present outbreak the owner was advised to submit his premises to thorough disinfection and to isolate rigidly all birds showing the slightest trace of illness. Since these measures were enforced only one further death occurred amongst the turkeys remaining on the premises.

\title{
THE METHYLENE-BLUE STAINING REACTION WITH ANTHRAX BLOOD.
}

\author{
By T. W. Cave, F.R.C.V.S., South-Eastern Agricultural \\ College, Wye, Kent.
}

FOR several years I have made frequent use of the methylene-blue staining reaction, described by Sir John M'Fadyean in the Journal of Comparative Pathology and Therapeutics for March 1903, in examining the blood in suspected cases of anthrax.

I have found the characteristic reaction to be perfectly reliable, and $I$ have had every confidence in basing my opinion on its presence or absence. There is, however, one peculiarity which, as far as I am aware, has not been previously recorded. When blood smears containing anthrax bacilli are stained with an aqueous solution of methylene-blue, the special reaction consists in a violet or reddish-purple staining of the amorphous material which is present between and around the bacilli. This colour reaction is obtainable in fresh blood, and may also still be seen in putrid blood after the anthrax bacilli have broken up and disappeared.

In the article to which I have referred, it is recommended that the stained film, after drying thoroughly, should be covered with Canada balsam and a cover-glass, and then examined under a microscope.

For a long time I have examined all blood smears, after staining and drying, without the use of a cover-glass, a method which, I think, has certain advantages.

A drop of cedar-wood oil is placed on the naked film, which is then examined with a $\frac{1}{12}$ inch objective. When anthrax bacilli are present the colour reaction is extremely vivid, and the amorphous material surrounding the bacilli presents a bright purplish-red colour.

If such a film is next covered with Canada balsam and a coverglass, and is examined from time to time, a remarkable change in the appearance of the film will presently be noticed. The vivid colouring is seen to be fading away, and sooner or later there is only 